\title{
Revisión de las Características de Sistemas de Almacenamiento de Energía para Aplicaciones en Micro Redes
}

\author{
Javier A. Guacaneme, David Velasco, César L. Trujillo
}

Universidad Distrital Francisco José de Caldas, Laboratorio de Investigación en Fuentes Alternativas de Energía, Facultad de Ingeniería, Carrera 7 № 40-53 Piso 5, Bogotá-Colombia

(e-mail: jguacaneme@udistrital.edu.co,davede@posgrado.upv.es, cltrujillo@udistrital.edu.co)

Recibido Ago. 26, 2013; Aceptado Oct. 7, 2013; Versión final recibida Oct. 23, 2013

\begin{abstract}
Resumen
Este artículo presenta una revisión de las principales tecnologías para almacenamiento de energía y su utilización en micro redes eléctricas. La utilización de fuentes de energía renovable en sistemas de distribución de energía eléctrica aumenta los requerimientos de instrumentación, gestión y control, debido al grado de intermitencia y variabilidad de dichas fuentes. Los sistemas de almacenamiento brindan la confiabilidad, disponibilidad y calidad del servicio que se requiere. Sin embargo, la selección del sistema de almacenamiento debe ser parte del diseño de la microred, teniendo en cuenta los objetivos y las características requeridas. El análisis muestra que la implementación de sistemas de almacenamiento de energía en una micro red mejora los transitorios, la capacidad, aumenta la potencia instantánea y permite la introducción de sistemas renovables de energía.
\end{abstract}

Palabras clave: sistemas almacenadores de energía, microred, energías renovables, integración energética

\section{Review of Characteristics of Energy Storage Systems for Microgrid Applications}

\begin{abstract}
This paper presents an overview of the main technologies for energy storage and use in electrical microgrids. Use of renewable energy sources in electric energy distribution increases instrumentation, management and control requirements, due to the high degree of intermittency and variability that these sources present. Storage systems provide reliability, availability and quality that are required. However, storage system selection must be part of microgrid design, taking into account the goals and the desired characteristics. The analysis shows that the implementation of energy storage systems on a microgrid improves transient response and capacity, increases instantaneous power and allows renewable energy integration.
\end{abstract}

Keywords: energy storage systems, microgrid, renewable energy, energy integration 


\section{INTRODUCCIÓN}

Una micro red es una red de energía a nivel de distribución, limitada en extensión con una capacidad menor a $100 \mathrm{~kW}$ (Lasseter, 2002), que incluye cargas, fuentes de energía distribuidas como generadores y dispositivos de almacenamiento de energía. Son sistemas totalmente autónomos gracias a la penetración de tecnologías de comunicación y control en el sistema de potencia, son consideradas como cargas para la red. Trabajan de dos modos, en conexión con la red y de manera aislada. Las micro redes ofrecen grandes oportunidades de integrar a pequeña escala, las fuentes de energía renovable a los sistemas de potencia local (Piagi y Lasseter, 2006).

Esta integración permite aumentar el porcentaje de energía proveniente de las fuentes renovables a la generación total de electricidad, por lo tanto se incrementa la sostenibilidad de la electricidad y en teoría aumenta la fiabilidad del sistema, la seguridad, la flexibilidad y la accesibilidad a la energía eléctrica (Dell y Rand, 2001; Bando et al., 2008). Sin embargo, las energías renovables son particularmente afectadas por parámetros externos como los medio ambientales, haciendo que este tipo de recurso sea inherentemente variable e intermitente en algunos casos (Bando, 2008). Un caso claro de este fenómeno se presenta cuando hay días nublados o sin brisa, lo que disminuye la producción de energía eléctrica proveniente de paneles fotovoltaicos y granjas eólicas, respectivamente. Además, la conexión de un gran número de fuentes de energía renovables puede ocasionar problemas de estabilidad al sistema de potencia local (Youli et al., 2009).

El almacenamiento convencional se concentra en las reservas hidráulicas en embalses o en sistemas de bombeo, sin embargo, la disponibilidad de sitios para su instalación es cada vez menor y se debe tener en cuenta el impacto ambiental. En este escenario sistemas de almacenamiento de energía de menor capacidad instalados de manera distribuida, pueden responder a las demandas actuales.

Con el fin de utilizar la energía renovable de manera adecuada, sin tener problemas relacionados con la variabilidad y la intermitencia de la energía, y la inestabilidad de la red eléctrica, debe ser diseñado e implementado un sistema de almacenamiento robusto el cual permite al sistema de potencia local interactuar con fuentes de energía renovables de diferentes características. La utilización adecuada puede llegar a ser competitiva a nivel técnico y económico en comparación con tecnologías basadas en combustibles fósiles o nucleares (Cavalho, 2001).

Debido a las diferencias propias de cada fuente de energía renovable actuante en una microred, se hace necesario el diseño de sistemas versátiles de almacenamiento de energía con capacidad de operar en amplios rangos de densidad de potencia y de densidad de energía. Dado que ninguna tecnología de almacenamiento de energía por si sola tiene esta capacidad, el sistema debe incorporar una combinación de diferentes tecnologías, como super condensadores, baterías, superconductores magnéticos de almacenamiento de energía o almacenamiento de energía cinética en volantes de inercia (Vázquez, et al., 2010).

La capacidad de un sistema de almacenamiento de energía depende de las características de compensación que se ofrecen. El tipo y la capacidad del sistema de almacenamiento empleado, son parámetros que deben ser seleccionados adecuadamente. En caso de una caída de tensión de corta duración, en la cual se pueden extraer corrientes muy altas en unos pocos ciclos, se debe usar un elemento con gran capacidad de potencia a costo de menor capacidad de almacenamiento de energía pero con una velocidad de respuesta alta. Sin embargo, si la caída de tensión se prolonga por un intervalo de tiempo mayor, tanto así que pueda ocasionar la interrupción del suministro eléctrico, se hace necesario el uso de fuentes adicionales de respaldo que permitan alimentar las cargas críticas.

En caso de eliminación de armónicos y compensación de energía reactiva, puede utilizarse un filtro pasivo adecuado, lo que influye en la disminución de la contribución del sistema de almacenamiento de energía (Bhatia, et al., 2005). Las mejoras en dispositivos semiconductores y la madurez de la electrónica de potencia, permiten interfaces con las características adecuadas para obtener los objetivos planteados con el almacenamiento.

\section{CONSIDERACIONES DEL ALMACENAMIENTO DE ENERGÍA}

La columna vertebral de un sistema energético se basa en la capacidad para generar energía suficiente, con el fin de atender la demanda a precios razonables y en condiciones medioambientales y de confiabilidad adecuadas. Por lo tanto, el almacenamiento de energía eléctrica ha sido un desafío en la implementación de sistemas energéticos, en razón a que diferentes factores afectan la generación de 
energía eléctrica, como son, la época del año (energía hidroeléctrica y eólica) y la intermitencia (solar), esto sin tener en cuenta los cambios de carga presentes en el sistema (Oudalov, et al., 2006).

Tabla 1. Clasificación de las aplicaciones de los sistemas de almacenamiento de energía.

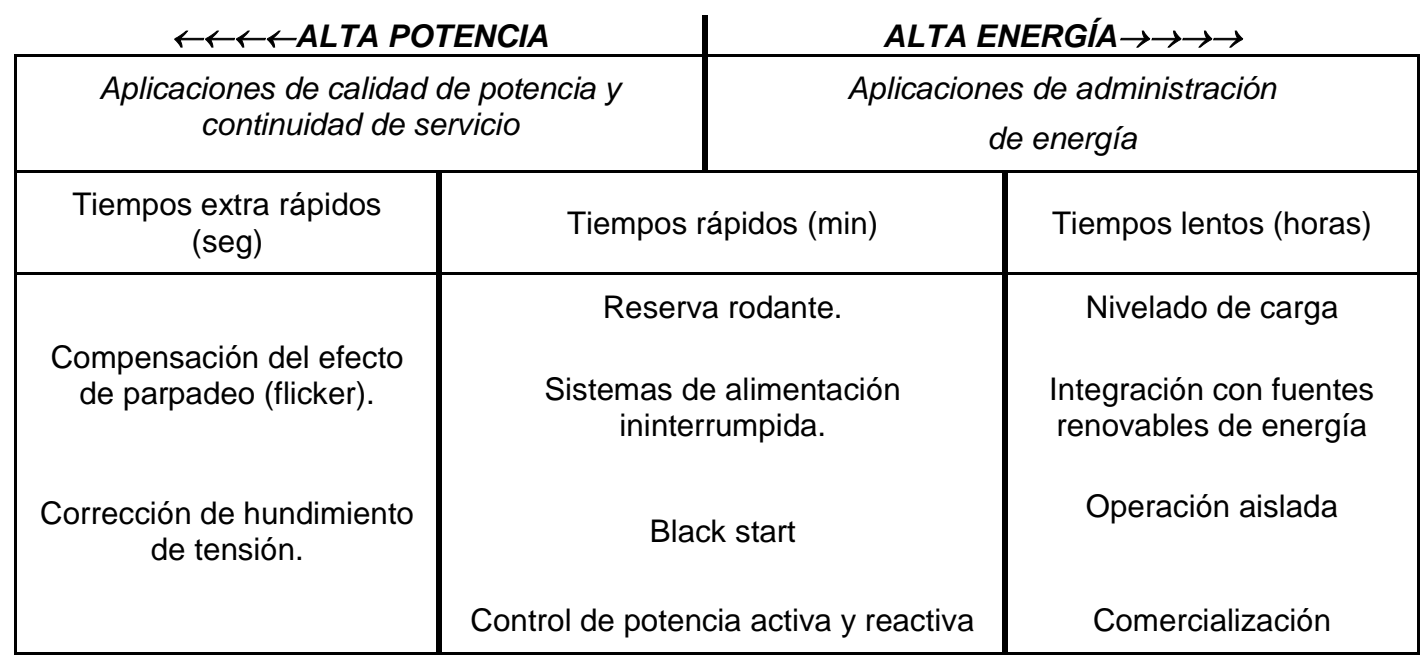

En la Tabla 1 se presenta una clasificación de las aplicaciones de los sistemas de almacenamiento de energía en los sistemas eléctricos de potencia. Dependiendo dela capacidad del sistema eléctrico, los requerimientos de los sistemas de almacenamiento cambian, es decir, si se trata de sistemas de alta potencia los sistemas de almacenamiento deben tener la capacidad de mantener la calidad de la energía y asegurar la continuidad del servicio (descargas de energía en fracciones de segundo), mientras que en los sistemas basados en generación distribuida la premisa es el almacenamiento de energía con el fin de entregarla en los momentos (horas) en los cuales la generación no está presente(Nourai, 2002).

En la actualidad existen diversas tecnologías que permiten el almacenamiento de energía eléctrica, como baterías, volantes de inercia, condensadores electroquímicos ("super" o "ultra" condensadores). El almacenamiento de aire comprimido, el almacenamiento térmico, y el bombeo de agua, son usualmente grandes con importantes requerimientos de instalación, y los superconductores magnéticos son dispositivos de almacenamiento de corta duración utilizados especialmente en aplicaciones de calidad de potencia. (Poopun y Jewell, 2008).

Se reconoce que beneficio principal del uso de sistemas de almacenamiento es la reducción del consumo de combustibles fósiles, sumando los beneficios técnicos que ofrecen las micro redes se mejoran los niveles de eficiencia de las unidades de generación también reducen las emisiones y permite diferir las inversiones en unidades de generación y en sistemas de transmisión.

Las micro redes son sistemas con baja inercia en contraste con los sistemas de generación convencionales, ante cambios de carga y suministro, la inercia de los sistemas sincrónicos y generadores de inducción entregan o absorben cualquier diferencia de potencia entre la generación y la demanda. En el modo de conexión a red la estabilidad depende de la habilidad de ajustar rápidamente la potencia activa o reactiva por medio de la electrónica de potencia, para controlar las oscilaciones de tensión o de frecuencia. Considerando a la red una fuente mucho más fuerte, los sistemas electrónicos de la microred se deben ajustar a las condiciones de operación de la red de manera transparente para la carga.

A continuación, se presentan los sistemas de almacenamiento de energía, más representativos utilizados en micro redes eléctricas, así como sus ventajas y desventajas.

\section{Baterías para sistemas de almacenamiento de energía}

Las baterías utilizadas en micro redes son las llamadas secundarias que permiten recargarse a partir de energía eléctrica revirtiendo el proceso electroquímico. El principio de funcionamiento de una batería es la reacción electroquímica entre dos electrodos separados por un electrolito como se muestra en la Fig. 1, las reacciones químicas en los componentes producen una corriente de iones en el electrolito que a su vez producen una corriente eléctrica en los electrodos. Las diferencias en las tecnologías de fabricación de baterías definen el costo, la capacidad, vida útil y tamaño entre otras. 


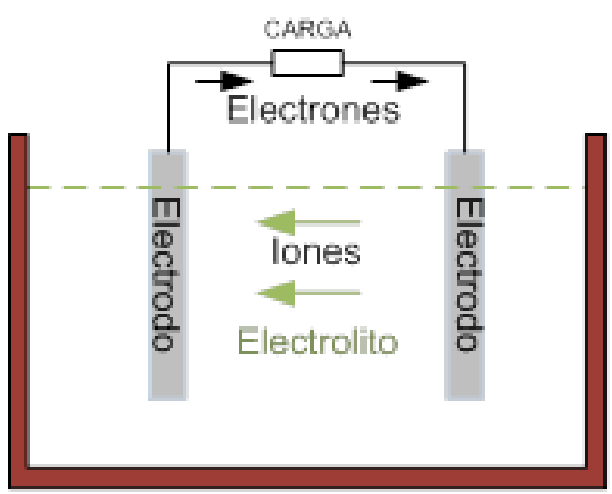

Fig.1. Batería electroquímica

La tecnología de plomo-ácido es la más madura con más de un siglo de desarrollo. Sus desventajas son la limitación en el número de ciclos de carga y descarga (alrededor de 1000), la contaminación debida al electrolito, y la densidad de energía que se traduce en peso y tamaño por lo que no son recomendables para sistemas que requieran movilidad o portabilidad. Gracias a su bajo costo, a una eficiencia cercana a $75 \%$ y la auto descarga moderada que presentan, han comprobado su eficacia en grandes sistemas de almacenamiento instalados en la década de 1980 a 1990, desde pequeños sistemas de KW hasta sistemas más grandes de algunas decenas de MW (Parker, 2001).

En comparación con la electroquímica del plomo-ácido, el níquel-cadmio ofrece una mayor densidad de energía, presenta un mayor número de ciclos de carga y descarga (cerca de 3500), con bajo mantenimiento. En Europa su uso está restringido a aplicaciones médicas específicas, es una tecnología de mayor costo de fabricación y contaminante por la toxicidad del cadmio. Otra tecnología de fabricación de baterías aún en desarrollo, es la de Níquel-metal hidruro, en reemplazo del Níquel-Cadmio, es una tecnología con menores costos de fabricación y que reduce la contaminación, con alta densidad de energía y ciclos de vida similares, reduciendo el efecto memoria. Sin embargo su desarrollo se ha estancado por el auge de las baterías de ión de litio.

Inicialmente se presentaron las baterías de ión de litio y posteriormente las de litio polímero, presentan la mayor densidad de energía (Hall y Bain, 2008), aunque su coste es alto se ha reducido por el aumento de producción y aceptación en el mercado de electrónica portátil de consumo. Las baterías de litio son la tecnología más prometedora en el campo de sistemas de almacenamiento de fuentes de energía renovables de pequeña escala a pesar de la necesidad de sistemas de carga complejos y a la falta de desarrollo de técnicas de gran escala.

Los sistemas de almacenamiento de energía basados en baterías utilizados para conexión a red, consisten en un banco de baterías provisto de sistemas de control y la interface de electrónica de potencia necesaria para acondicionar la energía eléctrica a los valores del sistema de distribución como en la Fig. 2. Los intercambios de potencia reactiva se pueden manejar por medio de los capacitores de filtrado de la electrónica, mientras que los de potencia activa definen el dimensionamiento de las baterías en función de la magnitud y la duración de la diferencia entre la potencia activa demandada por la carga y la potencia entregada por los generadores en la micro-red (Venkataramanan y Illindala, 2002).

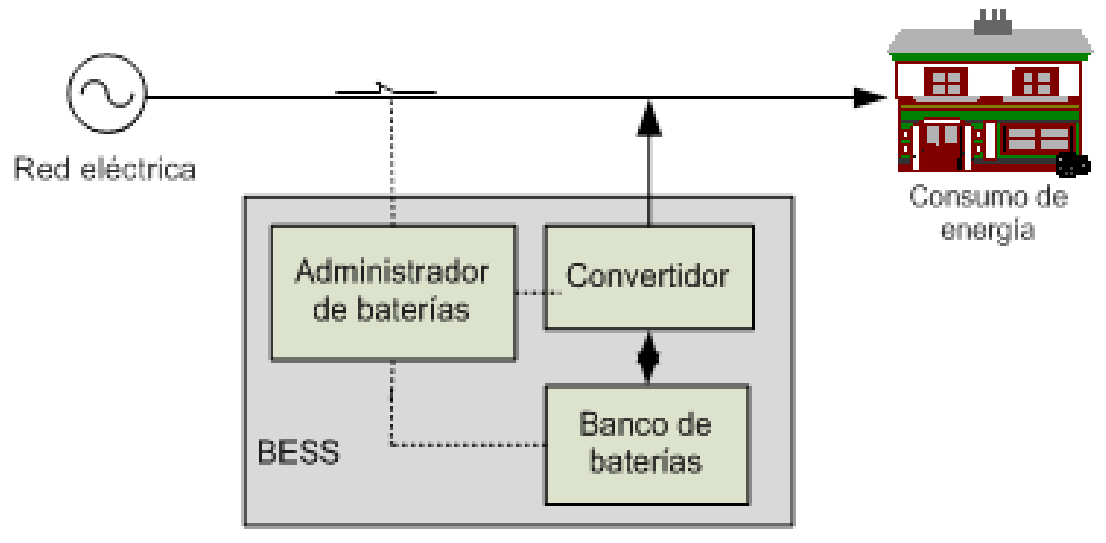

Fig. 2. Sistemas de almacenamiento de energía basados en baterías- BESS 
Ejemplo de una instalación se presenta en South Salem Oregon, un sistema de almacenamiento de baterías de ión de litio de $5 \mathrm{MW}$ desarrollado por Portland General Electric. En una microred de prueba del almacenamiento de energía eólica y solar, atiende a cerca de 500 usuarios comerciales y residenciales, hace parte del proyecto de demostración Pacific Northwest Smart Grid. Además de los beneficios en confiabilidad y calidad de potencia se tiene la capacidad de soportar la microred durante 30 minutos. Con la colaboración de los usuarios y un software especializado, permite almacenar energía cuando los precios del mercado son bajos y consumirla cuando aumentan.

\section{Baterías de flujo}

La principal característica de las baterías de flujo, es la independencia entre la capacidad de potencia y la energía que pueden manejar. La capacidad de almacenamiento depende exclusivamente de la cantidad de los electrolitos utilizados, mientras que la potencia depende del área activa de la celda. En la Fig. 3 se muestra un esquema de una célula de la batería de flujo.

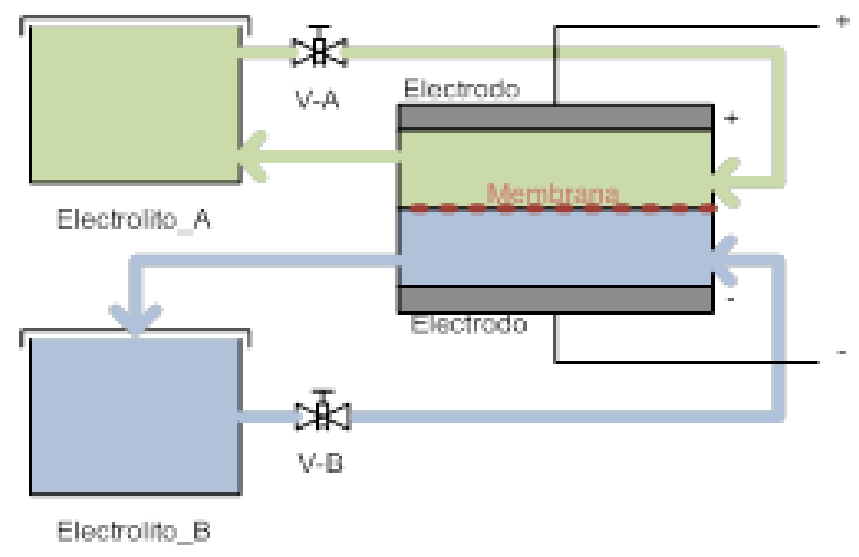

Fig. 3. Célula de la batería de flujo

Los electrolitos son almacenados en diferentes tanques y se hacen circular a través de la celda que los separa a través de una membrana microporosa que permite la corriente de iones, que a través de los electrodos, se transmiten como corriente eléctrica.

La reacciones que se producen son de reducción-oxidación, por lo cual también se conocen como baterías REDOX, las tecnologías de fabricación de las celdas de flujo en desarrollo son: Zinc-Bromuro, ZnBr; Reducción de Vanadio VRB; Zinc - Air, entre otros. Presentan una eficiencia entre el 50 y el $80 \%$, alta capacidad de potencia y energía, son de recarga rápida, los electrolitos no son tóxicos y pueden reemplazarse fácilmente, trabajan a bajas temperaturas y pueden tener una vida útil bastante alta. La desventaja de las baterías de flujo, es la movilidad de los electrolitos y la necesidad de bombeo con su correspondiente consumo de energía. Aunque su uso es limitado, se ha comprobado su funcionamiento en sistemas de capacidad de almacenamiento de 15MW-120MWh con la tecnología $\mathrm{VBr}$ con una eficiencia de cerca del 75\% (Couffin y Perrin, 2005).

En mayo de 2013 se inauguró una micro red en el fuerte Bliss, en Texas con un arreglo de $120 \mathrm{KW}$ de energía solar que incluye un sistema de almacenamiento de baterías de flujo de Zinc-bromuro, de $300 \mathrm{KW}$ construido por Primus, permite una descarga profunda del $100 \%$ sin daño de las baterías para una vida útil de 20 años. En esta aplicación también se pretende atender la carga que atiende a cerca de 8600 personas en ausencia de la red gracias a su capacidad de energía de cerca de 1 MWh.

\section{Supercondensadores}

Los condensadores electroquímicos de doble capa o supercondensadores presentan características de condensadores y de baterías, con la salvedad que su funcionamiento no depende de una reacción electroquímica, de esta manera el número de ciclos de carga y descarga es mucho mayor que en las baterías. La energía se almacena en los supercondensadores en forma de campo eléctrico entre dos electrodos, a diferencia de los condensadores. La superficie aislante del dieléctrico se reemplaza por un electrolito conductor de iones. En la Fig. 4 se muestra una célula del supercondensador ECDL (siglas del inglés Electrochemical Double Layer Capacitor). 


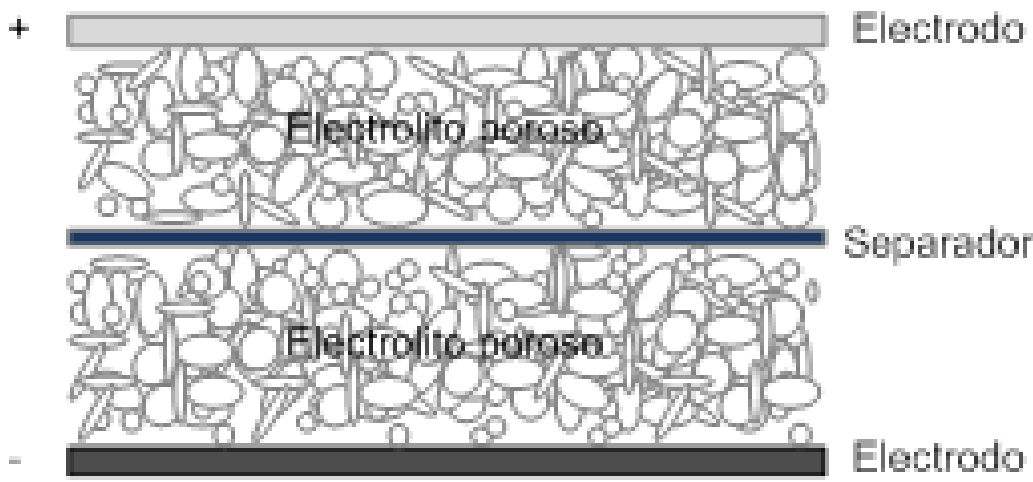

Fig. 4. Célula del supercondensador ECDL, (Burke, 2000)

La lentitud del movimiento de los iones a través del electrolito permite alcanzar mayores niveles de energía a costo de menor tensión (2.5 a 3V), a diferencia de los condensadores la conexión en serie es más común para alcanzar los niveles de tensión nominales de almacenamiento, la corriente que puede manejar es bastante alta debido a que la superficie de los electrodos es mucho mayor que la de los electrodos en condensadores. El tiempo de vida es mucho mayor que el de condensadores convencionales alcanzando más de 10 años (Bingbing, et al., 2010), con auto descargas de 5\% por día, es decir pueden utilizarse en aplicaciones rápidas.

Los supercondensadores responden a cargas y descargas muy rápidas, permitiendo descargas profundas y sobrecargas con una eficiencia energética de hasta el $98 \%$ debido a que no se presenta desgaste 0 calentamiento de los materiales. La densidad de potencia de los supercondensadores es bastante alta en contraste con la densidad de energía, debido a que su descarga es mucho más rápida que la de las baterías y presentan auto descargas del $14 \%$ por mes. Su alto costo reduce su penetración en usos comerciales, sin embargo, su uso combinado con baterías les permite extender su vida útil y reducir su capacidad mientras los supercondensadores responden a los picos de carga.

El objetivo de los supercondensadores en la microred es la mejora de la calidad de potencia, su gran respuesta en potencia pero por cortos periodos de tiempo lo hace especial para trabajar en conjunto con baterías o sistemas de almacenamiento con capacidad de energía. En el proyecto de Palmdale en California, la micro red consiste en $950 \mathrm{~kW}$ en turbinas eólicas, $200 \mathrm{~kW}$ en gas natural, $250 \mathrm{~kW}$ de turbinas hidráulicas, un sistema de almacenamiento de $450 \mathrm{~kW}$ y las cargas locales de $1.25 \mathrm{MW}$. El objetivo es mantener las condiciones de tensión, frecuencia, corrección de factor de potencia, mejora del contenido de armónicos, y reducción de transitorios.

\section{Celdas de combustible}

Este sistema se basa en el almacenamiento de energía en forma de combustible. Una celda de combustible es un dispositivo electroquímico, la electricidad se genera a partir de una fuente externa de combustible (habitualmente hidrógeno) y de oxígeno, sin combustión. Una celda de combustible no se agota ni requiere recarga. Producirá energía en forma de electricidad y calor mientras se le provea de combustible. El único subproducto que se genera es agua.(Boudghene, 2011). En la Fig. 5se muestra el esquema básico de la configuración del sistema.

El combustible necesario para la generación de energía en las celdas de combustible es el hidrógeno. Sin embargo, a pesar de su abundancia en la atmósfera, el hidrógeno no es de libre disposición y se produce principalmente en combinación con otros compuestos moleculares. La separación de hidrógeno a partir de estos compuestos es un proceso que demanda una gran cantidad de energía. La eliminación de hidrógeno a partir de combustibles fósiles es fácil ya que estos se encuentran en un estado de energía más alto. No obstante, tal proceso es altamente contaminante. La separación de hidrógeno a partir de agua necesita más energía, pero no libera ningún agente contaminante. El proceso de extracción de hidrógeno a partir de agua se llama electrólisis y se realiza a través de un electrolizador (Bajpai y Dash, 2012). 


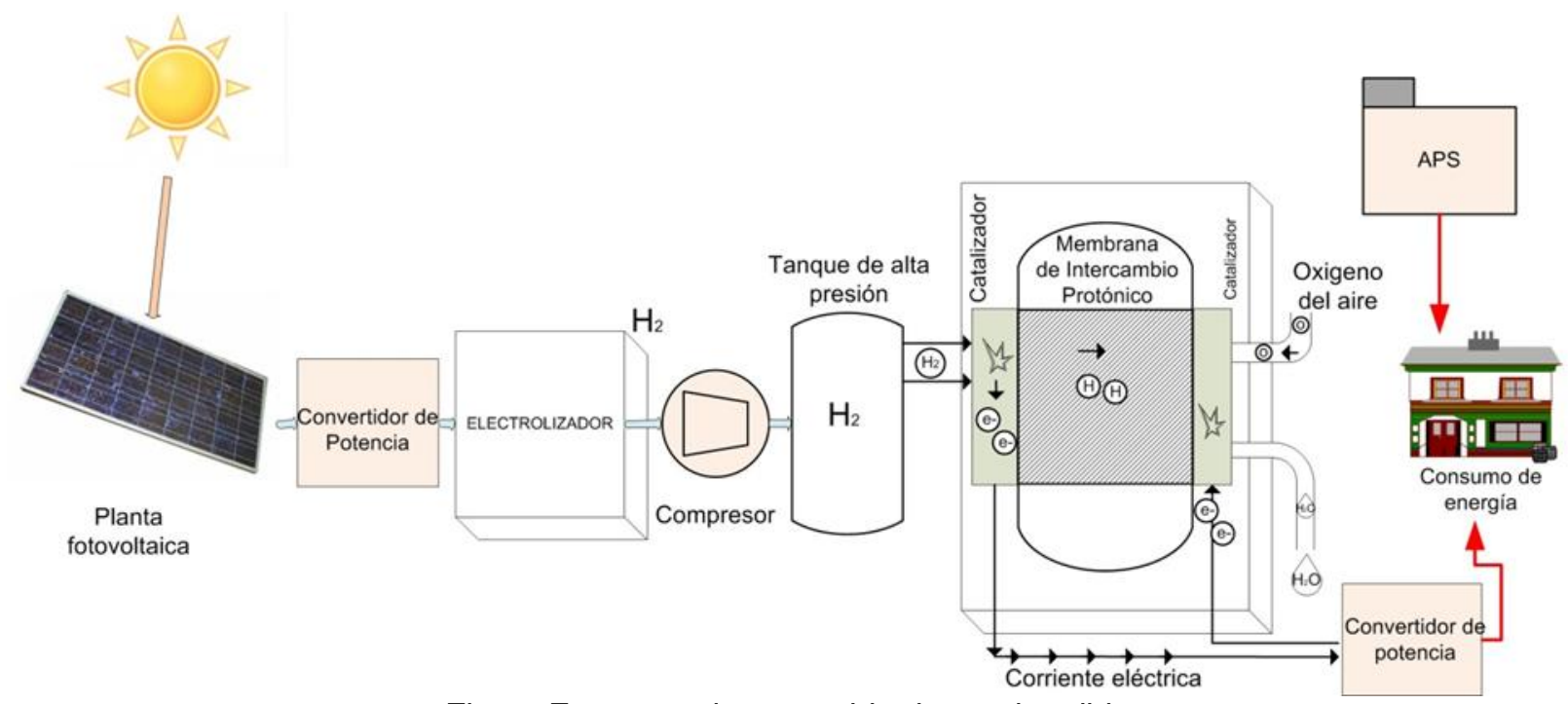

Fig. 5. Esquema de una celda de combustible

Los reactivos típicos utilizados en una celda de combustible son hidrógeno en el lado del ánodo y oxígeno en el lado del cátodo (si se trata de una celda de hidrógeno). La eficiencia total de una planta de proceso reversible (de electricidad a hidrógeno y de hidrógeno a electricidad), se encuentra en valores del $30-40 \%$. En el caso de centrales con cogeneración en la que se aprovecha el calor, el rendimiento se sitúa alrededor del $80 \%$. La respuesta de este sistema es lenta en comparación con las baterías. Entre todos los tipos de celdas de combustible, la celda de combustible de membrana de intercambio protónico es muy prometedora debido a su funcionamiento a baja temperatura y la rápida puesta en funcionamiento.

Ejemplo del uso de celdas de combustible es la micro red de la cárcel Santa Rita en el condado de Alameda en California, con una capacidad de 4000 personas, la celda de hidrógeno de $1 \mathrm{MW}$, provee de calidad de potencia al sistema y adicionalmente cogeneración para calentamiento de agua y aire acondicionado. Reduce el consumo de energía del alimentador en cerca de un $80 \%$ de la demanda pico en los meses de verano.

\section{Sistemas de almacenamiento de energía en supercondutores magnéticos - (SMES)}

Los sistemas SMES (siglas del inglés Superconducting Magnetic Energy Storage) almacenan energía eléctrica en un campo magnético creado por un flujo de corriente continua en una bobina superconductora. Esta bobina superconductora es refrigerada criogénicamente a una temperatura inferior a la temperatura critica de superconducción (Nagaya, et al., 2012). Un sistema SMES suele estar compuesto por la bobina superconductora, el sistema de acondicionamiento de potencia y el refrigerador criogénico como se representa en la Fig. 6.

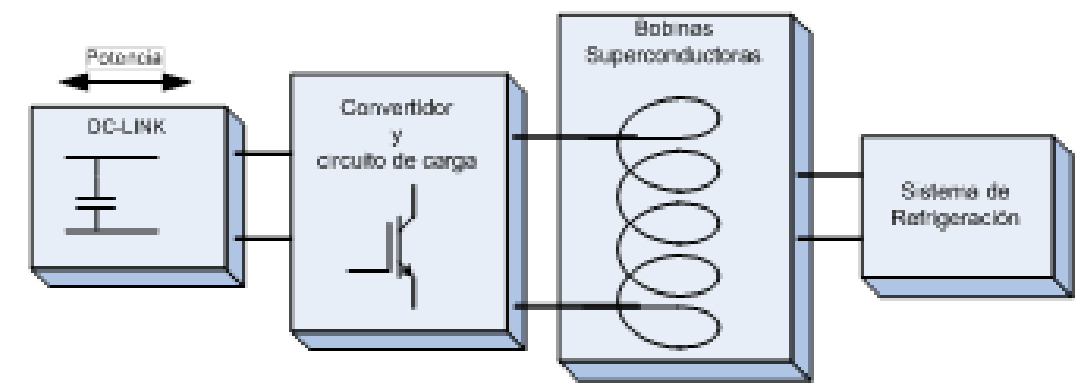

Fig. 6. Esquema de sistema de almacenamiento de energía en superconductores magnéticos

La energía almacenada puede ser devuelta a la red mediante la descarga de la bobina. El sistema de acondicionamiento de potencia suele estar compuesto por un inversor bidireccional que permite sacar energía de la red hacia la bobina e inyectar la energía de la bobina en la red. Estos sistemas también pueden ser empleados conectados a una línea de alterna mediante inversores o a líneas de corriente continua.

Las pérdidas del sistema de acondicionamiento de energía suelen estar en torno al 2-3\% en cada dirección por lo que teniendo en cuenta la carga y la descarga, la eficiencia total del sistema será mayor del $95 \%$. Una de las ventajas de estos sistemas es que pueden ser descargados prácticamente por completo, contrario a 
las baterías (Kim, et al., 2012). Tiene una elevada velocidad de respuesta (por debajo de los $100 \mathrm{~ms}$ ) lo que los hace óptimos para regular la estabilidad de la microred. La principal desventaja es la refrigeración.

Se logran almacenar grandes cantidades de energía (5000 a 10000MWh) requiriendo bobinas de gran tamaño y se precisa su instalación bajo tierra para minimizar costos de infraestructura (Shi, et al., 2010). La aplicación en micro redes aún es incipiente, la fuerza aérea de los Estados Unidos y su programa Power Continuation Integration Equipment, construyó en 1996 sistemas micro SMES de 6 MJ, 750 KVA para aplicaciones de calidad de potencia principalmente interrupciones y huecos de tensión, con una capacidad de $454 \mathrm{KW}$ durante 9 segundos y una vida útil de 20 años. En la ciudad de Kameyama, Mie, en Japón, se encuentra instalado en el sistema de potencia un SMES de 10 MVA y $20 \mathrm{MJ}$, con excelentes resultados en la estabilización del sistema con altos estándares de calidad de potencia.

\section{Volantes de inercia - (FES)}

Los volantes de inercia FES (siglas del inglés Flywheel Energy Storage), almacenan energía mediante la aceleración de un rotor (volante) a una velocidad muy alta y la energía se mantiene en el sistema como energía de rotación. Cuando la energía se extrae del sistema, la velocidad de rotación del volante se reduce como consecuencia del principio de conservación de la energía. Al inyectar energía al sistema resulta en un aumento de la velocidad del volante. El sistema consiste en un rotor suspendido por rodamientos (magnéticos en los grandes sistemas) y en un recinto al vacío para reducir el rozamiento (Bolund, et al., 2007). En la Fig.7 se muestra un esquema de un sistema FES.

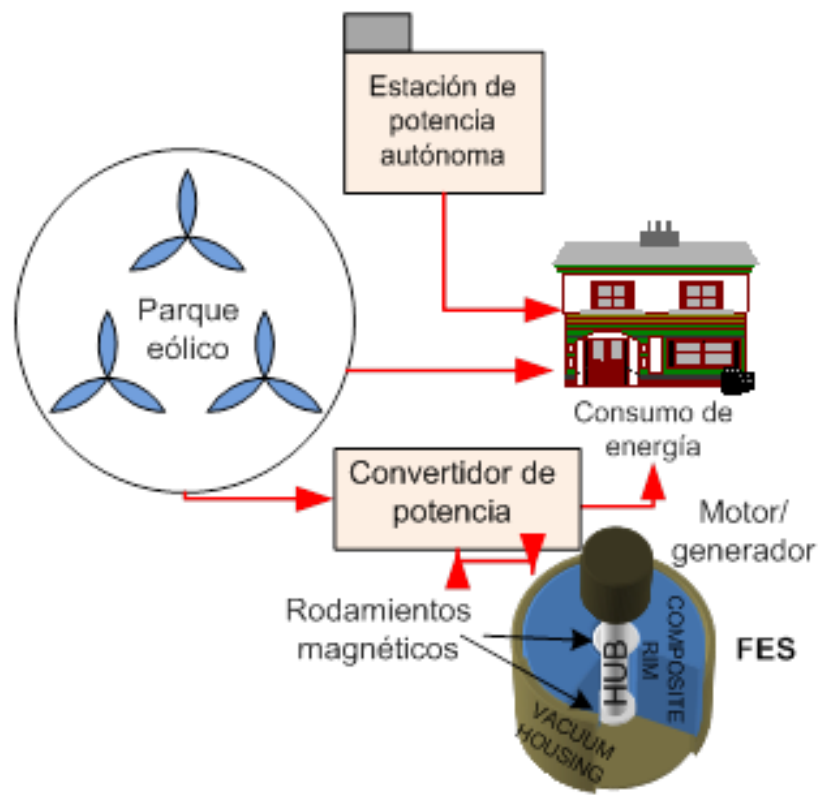

Fig. 7. Esquema de un volante de inercia

Los sistemas FES avanzados tienen rotores hechos de fibra de carbono de alta resistencia, suspendido por los campos magnéticos. La velocidad de giro del volante va de 20.000 a más de 100.000 rpm en recintos al vacío. Estos volantes pueden llegar a la velocidad máxima en cuestión de minutos, mucho más rápido que algunas otras formas de almacenamiento de energía (Bai, et al., 2011). La eficiencia de estos dispositivos puede llegar al 90\% (Hittinger, et al., 2011).

Se trata de sistemas de respuesta rápida capaces de almacenar y liberar mucha energía rápidamente. Un ejemplo de una micro red que dispone de un volante de inercia de $100 \mathrm{~kW}$ es la implementada por la Universidad de Manchester (UK) (Lidula y Rajapakse, 2011).La micro red de prueba de 20 KVA, cargas de $12 \mathrm{KW}$, un generador de $22 \mathrm{~kW}$ y el SMES que almacena $4 \mathrm{MJ}$, ha demostrado suministro de potencia con altos niveles de calidad de potencia.

\section{Almacenamiento de energía mediante aire comprimido - (CAES)}

Los sistemas CAES (siglas del inglés Compressed Air Energy Storage), utilizan la energía comprimida asociada al aire presurizado contenido en depósitos subterráneos, consistente en cavidades naturales o antiguas minas o en acuíferos porosos o en depósitos construidos por el hombre. El almacenamiento se realiza comprimiendo el aire durante horas de baja demanda de energía eléctrica. Durante horas de alta 
demanda, el aire comprimido se calienta y expande a través de una turbina de combustión para generar energía eléctrica, con una alta eficiencia relativa. Si bien es cierto que este proceso requiere ciclo combinado, el consumo de gas natural para calentar el aire comprimido es menor que si se usaran las centrales de gas convencionales para generar electricidad (Ribeiro, et al, 2001).

Hoy en día las instalaciones más comunes de CAES van desde los 35MW hasta los 300MW con una eficiencia entre $70 \%$ y $80 \%$ (Vázquez, et al., 2010). Tienen la ventaja de poder utilizarse con sistemas a gran escala y poseer periodos de almacenamiento largos debido al hecho de que las pérdidas son muy pequeñas.

Una instalación típica de CAES se presenta en la Fig. 8. Se puede utilizar para almacenar energía durante más de un año. Proporciona tiempos de arranque rápidos (9 minutos para un arranque de emergencia, y aproximadamente 12 minutos bajo condiciones normales), en comparación con la turbina de combustión convencional, la cual en horas pico, puede requerir 20-30 minutos para un inicio normal (Hadjipaschalis, et al., 2009). Su principal desventaja es que se deben construir o adecuar en donde las condiciones geológicas del terreno lo permitan y sus altos costos de inversión no permiten que sean una solución para implementación a pequeña escala (Alamri y Alamri, 2009; Chouhan y Ferdowsi, 2009).

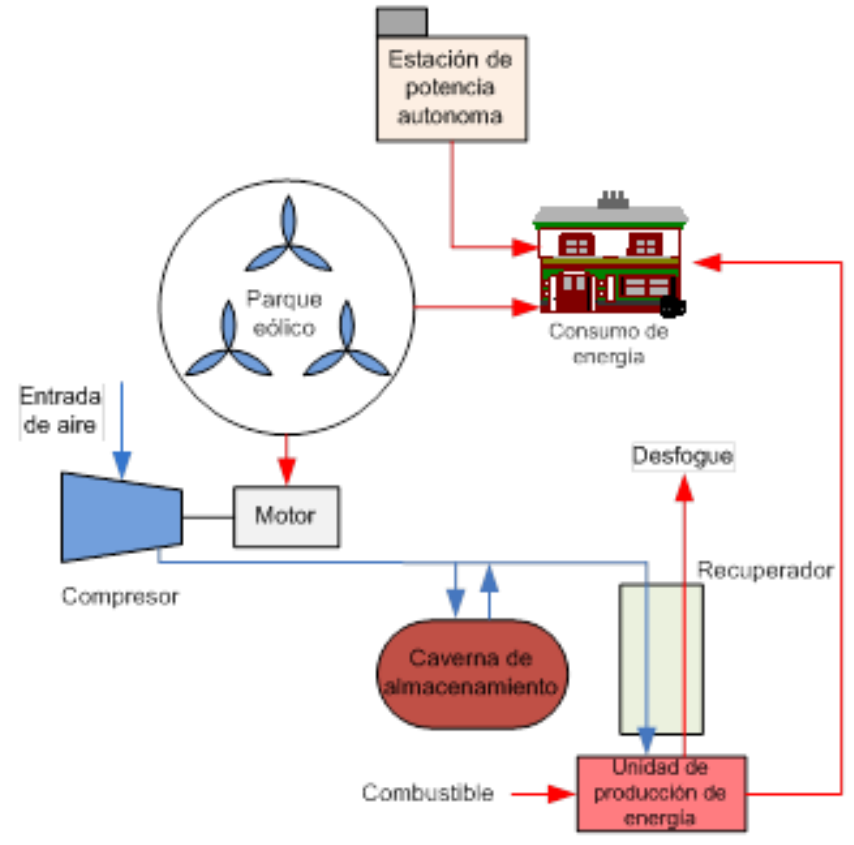

Fig. 8: Esquema de almacenamiento de energía mediante aire comprimido

Existen 2 sistemas CAES de gran escala en funcionamiento. Uno en Alemania en Huntorf, con una capacidad de carga $60 \mathrm{MWel}$ (8horas), y de descarga de $321 \mathrm{MWel}$ (2 horas). La otra ubicada en Mclntosh, Alabama USA, tiene una capacidad de carga de $60 \mathrm{MWel}$ (45 horas), y de descarga de $110 \mathrm{MWel}$ (26 horas).Ejemplo a baja escala es el presentado por la empresa SustainX del sistema isotérmico ICAES, en New Hampshire, Ohio USA, de 1.5 MW y una vida útil de 20 años, su implementación con micro redes es parte de proyectos futuros, como los presentados con energía eólica en el estado de Washington, USA, uno en Columbia Hills, con capacidad de $207 \mathrm{MW}$, almacenamiento de $231 \mathrm{MW}$ para almacenamiento continuo por 40 días y otro en Yakima Minerals Site con capacidad de 83 MW y capacidad de almacenamiento de $150 \mathrm{MW}$.

\section{Sistema de almacenamiento basado en bombeo hidráulico- (PHS)}

En un sistema de bombeo hidráulico de almacenamiento PHS (siglas del inglés Pumped Hydro Storage), el excedente de energía que aparece en los momentos de baja demanda y aumento de la producción (por ejemplo, el proveniente de los parques eólicos o las estaciones PV existentes) se aprovecha para bombear agua hasta un deposito ubicado a mayor altura, para almacenarla en forma de energía potencial, como se muestra en la Fig. 9. En consecuencia, durante períodos de máxima demanda, el agua se libera desde el depósito, accionando las turbinas hidráulicas, las cuales se encuentran acopladas a un generador, el cual permite producir energía eléctrica. Los PHS son capaces de cubrir el déficit de energía existente (capacidad de respuesta rápida) de acuerdo con la cantidad de energía almacenada. 


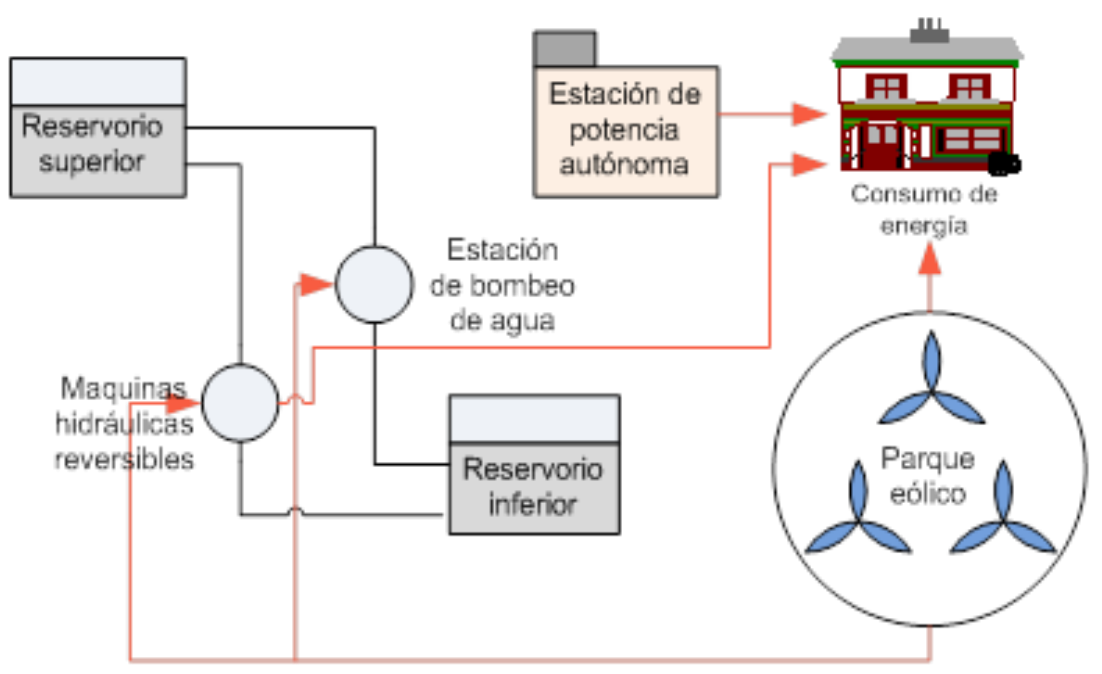

Fig. 9. Sistema de almacenamiento basado en bombeo hidráulico

La eficiencia global típica de estos sistemas oscila entre el 70\% y el 85\% (Ibrahima, et al., 2008), mientras que la profundidad máxima de descarga puede llegar a un 95\% sin afectar de forma significativa el tiempo de servicio (aproximadamente 50 años). El principal problema para la implementación de un PHS es que requiere generalmente de dos depósitos ubicados en cotas diferentes (aprox. $100 \mathrm{~m}$ ) lo que acarrea un alto coste de capital. Otro factor a tener en cuenta, es el impacto ambiental causado durante la construcción de la obra y la operación en los alrededores de la misma (Denholm y G.L. Kulcinski, 2004).

A pesar de los altos costos asociados de construcción, los costos de operación y mantenimiento son bajos, los PHS tienen la capacidad de brindad estabilidad a la red eléctrica debido a la rápida capacidad de respuesta ante repentinos cambios de carga contrario a la respuesta que presentan las centrales térmicas (Hadjipaschalis, et al., 2009). Actualmente, los PHS son los esquemas de almacenamiento de energía más rentables.

Ejemplo de aplicación es la micro red en la isla del Hierro en España, obteniendo 100\% de su energía de fuentes renovables con 11.5 MW de eólica, 11.3 MW de PHS con una estación de bombeo de $6 \mathrm{MW}$, la isla tiene $278 \mathrm{Km}^{2}$ de 10500 habitantes una demanda pico de 7MW y de $40 \mathrm{GWh} /$ año.

\section{Almacenamiento de energía térmica - (TES)}

Existen dos formas de almacenar energía térmica, dependiendo de si se utiliza el calor sensible o el calor latente. Los TES (siglas del inglés Thermal Energy Storage), basados en calor latente, hacen uso de la transición líquido-sólido de un material a temperatura constante. Durante la acumulación, el material se desplaza desde el estado sólido al líquido y, durante la recuperación, se pasa de líquido al sólido. Las transferencias de calor entre el acumulador térmico y el ambiente exterior se realizan a través de un fluido de transferencia de calor. La energía se almacena a una temperatura dada, cuanto mayor sea la temperatura más alta es la concentración, es decir la entalpía de fusión aumenta con la temperatura de fusión del material utilizado. A pesar de su naturaleza altamente corrosiva, el hidróxido de sodio se considera un buen fluido de almacenamiento (Ibrahima, et al., 2008).

Los TES basados en calor sensible, utilizan un aceite sintético o sales fundidas que almacenan energía en forma de calor, el cual es colectado por las centrales eléctricas termo-solares, para producir vapor de agua a fin de accionar un sistema turbo-alternador, y de esta manera permitir la entrega de potencia de forma suave durante períodos nublados del día y adicionalmente, ampliar la producción de energía por espacio de 1 a 10 horas, después de la puesta de sol (Oudalov, et al., 2008).

Otro uso, consiste en almacenar la electricidad sobrante en almacenadores de calor o frio, tanques de hielo, depósitos de agua u otros materiales con el fin de reducir el consumo de electricidad mediante su empleo en sistemas de calefacción o sistemas de aire acondicionado, aumentando de esta forma la capacidad de generación en momentos de demanda pico. En la Fig. 10se puede observar un diagrama de un TES. 


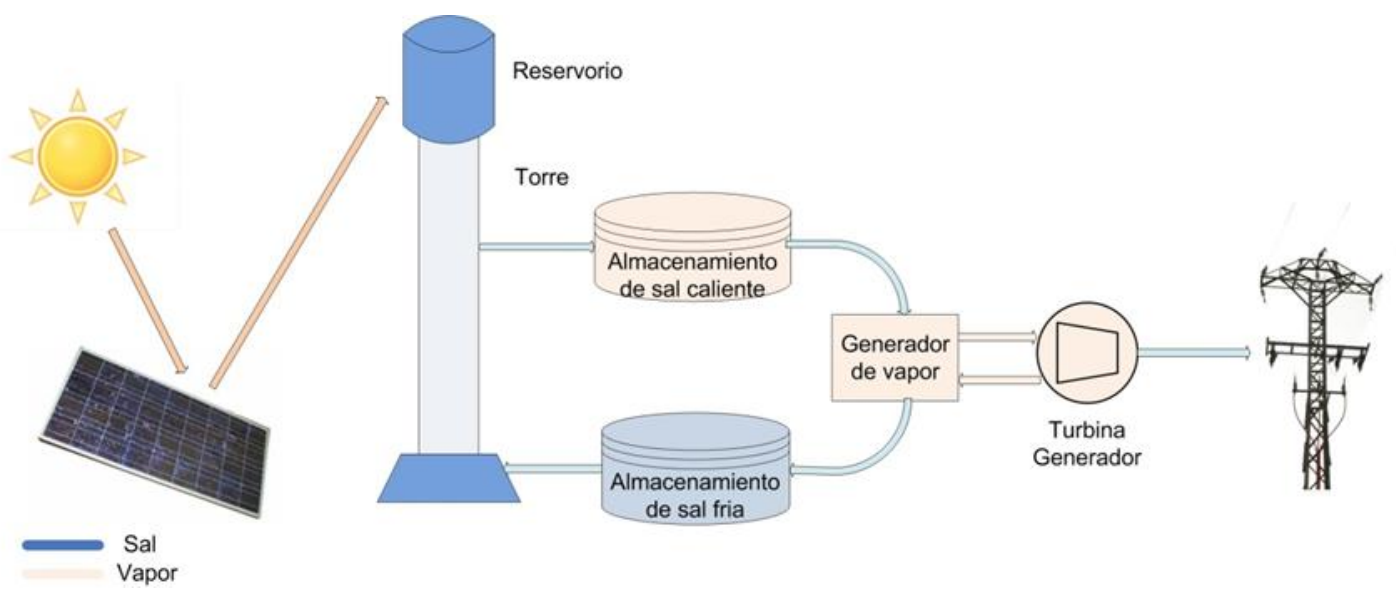

Fig. 10. Esquema de almacenamiento de energía térmica

Ejemplo del almacenamiento con TES se encuentra en la Universidad del sur de California con tanques de 4.5 millones de galones / 60000 ton-horas), incluye energía solar de $1 \mathrm{MW}$, gas natural de ciclo combinado de $19 \mathrm{MW}$. Se permite atender el $65 \%$ de la carga durante el día recargando se durante la noche.

\section{Comparación de eficiencia, energía y potencia de los sistemas de almacenamiento de energía}

Determinar el componente básico del almacenamiento en una microred debe considerar el uso conjunto de dos o más tecnologías. Las características complementarias de velocidad de respuesta y capacidad de potencia o energía, permitirán atender las necesidades del almacenamiento en la integración y penetración de fuentes alternativas de energía con la red, proporcionando al mismo tiempo los requerimientos de estabilidad y de calidad de potencia.

En la Tabla 2 se resumen las características más relevantes de las tecnologías de almacenamiento de energía analizadas. Aunque realizar una comparación en términos absolutos es imposible debido a la diferente naturaleza del almacenamiento de energía, se presenta como información base de análisis. Las implicaciones de acceso a la tecnología, operación y mantenimiento, así como los requerimientos de funcionamiento temperatura, velocidad, tiempos de arranque, etc. deberán determinarse según los objetivos que se pretenden en la microred.

Tabla 2. Comparación de tecnologías para sistemas de almacenamiento de energía

\begin{tabular}{|c|c|c|c|c|c|}
\hline Tecnología & $W h / K g$ & Wh/litro & Potencia W/kg & $\begin{array}{c}\text { Tiempo medio } \\
\text { de descarga }\end{array}$ & Eficiencia \\
\hline Plomo-Ácido & $30-40$ & $60-75$ & 180 & $\begin{array}{c}10 \text { sec- varias } \\
\text { horas }\end{array}$ & $50-92 \%$ \\
\hline Ion-Li & $100-265$ & $250-730$ & $250-350$ & $\begin{array}{c}15 \text { min- varias } \\
\text { horas }\end{array}$ & $80-90 \%$ \\
\hline Níquel metal hidruro & $60-120$ & $140-300$ & $250-1000$ & $\begin{array}{l}\text { Autodescarga } \\
\text { de } 30 \% / \text { mes }\end{array}$ & $66 \%$ \\
\hline Sulfuro de Sodio & 100 & 150 & 200 & 8 horas & $70 \%$ \\
\hline Redox & $\sim 40$ & -- & $80-150$ & -- & $80 \%$ \\
\hline Supercondensadores & $3-5$ & -- & 6000 & $\begin{array}{c}\text { Autodescargas } \\
\text { Hasta } 5 \% \text { / día } \\
\text { Hasta } 14 \% \text { / mes }\end{array}$ & $95 \%$ \\
\hline $\begin{array}{c}\text { Celdas de } \\
\text { Combustible }\end{array}$ & 121 & 16 & 18 & -- & $80 \%$ \\
\hline SMES & $<1$ & -- & 1000 & $\begin{array}{c}\text { Menos de } \\
100 \mathrm{~ms}\end{array}$ & $90 \%$ \\
\hline $\begin{array}{c}\text { Volantes de inercia } \\
\text { FES } \\
\end{array}$ & $11-50$ & -- & $180-1800$ & $\begin{array}{c}20 \% \text { a } 50 \% \text { en } \\
2 \text { horas } \\
\end{array}$ & $90-95 \%$ \\
\hline CAES & $10-30$ & -- & -- & -- & $>60 \%$ \\
\hline PHS & 0.3 & -- & -- & -- & $70-85 \%$ \\
\hline TES & -- & 177 & -- & -- & $>60 \%$ \\
\hline
\end{tabular}




\section{CONCLUSIONES}

La debilidad de los sistemas de distribución de potencia eléctrica es la capacidad de almacenamiento, especialmente cuando la fuente de energía es intermitente y aislada de los centros de consumo, como en el caso de las micro redes. Tras realizar un análisis de los mismos se presentan diferentes ventajas e inconvenientes de cada uno de ellos de lo que se desprende que no existe un sistema óptimo. Lo que podría considerarse como óptimo es una combinación de diferentes sistemas aprovechando sus características de velocidad de respuesta, capacidad de almacenamiento o densidad energética. Esta elección debe realizarse con base en las características de la microred como sus fuentes de generación, sus cargas y el patrón de consumo.

De la implementación de sistemas de almacenaje de energía en una microred se obtienen ventajas como la mejora de los transitorios, mejora de la capacidad, aumento de potencia instantánea, al mismo tiempo que permite la introducción de sistemas renovables de energía.

Hay costos asociados al almacenamiento de energía, pero la administración en conjunto en sistemas como las micro redes genera beneficios técnicos y reducción del coste de la energía al consumidor. La integración de nuevas fuentes de energía sólo puede ser posible con sistemas que permitan la administración atemporal de la energía eléctrica con independencia de la naturaleza y ocurrencia de la fuente y la carga.

Las diferentes ofertas de almacenamiento cada una con diferentes características, hacen difícil una comparación objetiva, sin embargo es necesario presentar la variedad para, según los requerimientos, adaptarla a las necesidades propias de cada micro red.

\section{REFERENCIAS}

Alamri, B.R. y A.R. Alamri, Technical review of energy storage technologies when integrated with intermittent renewable energy, International Conference on Sustainable Power Generation and Supply. SUPERGEN '09, 1 - 5, Nanjing, China, 6 y 7 de Abril (2009).

Bai, J.G., X.Z. Zhang, y L.M. Wang, A Flywheel Energy Storage System with Active Magnetic Bearings, Energy Procedia, 16 (B), 1124-1128, (2012).

Bajpai, P. y V.Dash, Hybrid renewable energy systems for power generation in stand-alone applications: $A$ review, Renewable and Sustainable Energy Reviews, 16 (5), 2926-2939, (2012).

Bando, S., Y. Sasaki,H. Asano, y S. Tagami,Balancing control method of a microgrid with intermittent renewable energy generators and small battery storage, Power and Energy Society General Meeting Conversion and Delivery of Electrical Energy in the 21st Century, 1-6, Pittsburg, USA, 20-24 de Julio, (2008).

Bhatia, R.S., S.P. Jain, D.K. Jain, y B. Singh, Battery Energy Storage System for Power Conditioning of Renewable Energy Sources, International Conference on Power Electronics and Drives Systems, 2005. PEDS 2005, 1, 501-506, Kuala Lumpur, Malasia, (2005).

Bingbing, W., Y. Zhongdong, y X. Xiangning, Super-capacitors energy storage system applied in the microgrid, IEEE Conference on Industrial Electronics and Applications (ICIEA), 1002-1005, Taichung, Taiwan, 15 al 17 de Junio, (2010).

Bolund, B., H. Bernhoff, y M. Leijon, Flywheel energy and power storage systems, Renewable and Sustainable Energy Reviews, 11 (2),235-258, (2007).

Boudghene, A., Fuel cells: The expectations for an environmental-friendly and sustainable source of energy, Renewable and Sustainable Energy Reviews, 15 (9), 4507-4520, (2011).

Burke A., Ultracapacitors: Why, how, and where is the technology, Journal of Power Sources, 37-50, (2000)

Cavalho, A.J., Energy Storage Technologies for Utility Scale Intermittent Renewable Energy Systems, Journal of Solar Energy Engineering, 123 (4), 387-389, (2001).

Chouhan, N. y M. Ferdowsi, Review of energy storage systems, North American Power Symposium (NAPS), 1- 5,Starkville, USA, 4 a 6 de Octubre (2009).

Couffin, P. y M. Perrin, Le stockage des énergies intermittentes, Commissariat á l'Energie Atomique (CEA). Clefs CEA (50-51), www.cea.fr, 136-138, (2005). 
Dell, R.M. y D.A.J. Rand, Energy storage-a key technology for global energy sustainability, Journal of Power Sources, 100 (2), 2-17, (2001).

Denholm, P. y G.L. Kulcinski, Life cycle energy requirements and greenhouse gas emissions from large scale energy storage systems, Energy Conversion and Management, 45, 2153-2172, (2004).

Hadjipaschalis, I., A.Poullikkas, y V.Efthimiou, Overview of current and future energy storage technologies for electric power applications, Renewable and Sustainable Energy Reviews, 13(6-7). 1513-1522, (2009).

Hall, P.J. y E.J. Bain, Energy storage technologies and electricity generation, Energy Policy, 36, 4352-4355, (2008).

Hittinger, E., J.F. Whitacre, y J. Apt., What properties of grid energy storage are most valuable?, Journal of Power Sources, 206, 436-449, (2012).

Ibrahima, H., A. Ilincaa, y J. Perronb, Energy storage systems-Characteristics and comparisons, Renewable and Sustainable Energy Reviews, 12 (15), 1221-1250, (2008).

Kim A., y otros 5, SMES application for frequency control during islanded microgrid operation, Physica C: Superconductivity,484, 282-286, (2013).

Lasseter, R.H., "MicroGrids," Power Engineering Society Winter Meeting, 2002. IEEE, 1(1), 305-308, (2002). Lidula, N.W.A. y A.D. Rajapakse, Microgrids research: A review of experimental microgrids and test systems, Renewable and Sustainable Energy Reviews, 15 (1), 186-202, (2011).

Nagaya, S. y otros 7, The state of the art of the development of SMES for bridging instantaneous voltage dips in Japan, Cryogenics, 52 (12), 708-712, (2012).

Nourai, A., Large-scale electricity storage technologies for energy management, Power Engineering Society Summer Meeting 2002,Chicago, USA, 25 de Julio, (2002).

Oudalov, A., T. Buehler, y D. Chartouni, Utility scale applications of energy storage, IEEE Energy Conference, 1-7, Atlanta, USA,17-18 de Noviembre, (2008).

Oudalov, A., D. Chartouni, C. Ohler, y G. Linhofer, Value analysis of battery energy storage applications in power systems, Power Systems Conference and Exposition 2006 (PSCE'06), Atlanta, USA, 29 Oct.,(2006).

Parker, C.D., Lead-acid battery energy-storage systems for electricity supply networks, Journal of Power Sources, 100 (1-2), 18-28. (2001).

Piagi, P. y R.H. Lasseter, Autonomous control of microgrids, IEEE Power Engineering Society General Meeting, 1-8, Montreal, Canadá,(2006).

Piyasak P. y T. J. Ward, Analysis of the Cost per Kilowatt Hour to Store Electricity IEEE Transactions on Energy conversion, 23 (2), 529-534,(2008)

Ribeiro, P. F., B. K. Johnson, M. L. Crow, A. Arsoy e Y. Liu, Energy storage systems for advanced power applications, Proceedings of the IEEE, 89 (89), 1744-1756, (2001).

Shi, J., Y. Tang, T. Dai, L. Ren, J. Li, y S. Cheng, Determination of SMES capacity to enhance the dynamic stability of power system, Physica C: Superconductivity, 470 (20), 1707-1710, (2010).

Vázquez, S., S.M. Lukic,E. Galvan, L.G. Franquelo,y J.M. Carrasco, Energy Storage Systems for Transport and Grid Applications, IEEE Transactions on Industrial Electronics, 57 (12), 3881-3895, (2010).

Venkataramanan, G. y M. Illindala, Microgrids and sensitive loads, Power Engineering Society Winter Meeting, 2002. IEEE , 1(1), 315-322, (2002)

Youli, S., Z. Litifu, y K. Nagasaka, Efficiency of Micro Grid with Storage Battery in Reliability, Economy and Environment Assessments, International Journal of Electrical and Power Engineering, 3 (3), 154-162, (2009). 
